\title{
BMJ Open Subarachnoid haemorrhage guidelines and clinical practice: a cross-sectional study of emergency department consultants' and neurospecialists' views and risk tolerances
}

\author{
J Lansley, ${ }^{1,2}$ C Selai, ${ }^{3}$ A S Krishnan, ${ }^{2}$ K Lobotesis, ${ }^{4}$ H R Jäger ${ }^{5}$
}

To cite: Lansley J, Selai C, Krishnan AS, et al. Subarachnoid haemorrhage guidelines and clinical practice: a cross-sectional study of emergency department consultants' and neurospecialists' views and risk tolerances. BMJ Open 2016;6:e12357.

doi:10.1136/bmjopen-2016012357

- Prepublication history and additional material is available. To view please visit the journal (http://dx.doi.org/ 10.1136/bmjopen-2016012357).

Received 20 April 2016 Revised 10 July 2016 Accepted 16 August 2016

CrossMark

For numbered affiliations see end of article.

Correspondence to Dr J Lansley;

dr.joseph.lansley@gmail.com

\section{ABSTRACT}

Objectives: To establish if emergency medicine and neuroscience specialist consultants have different risk tolerances for investigation of suspected spontaneous subarachnoid haemorrhage (SAH), and to establish if their risk-benefit appraisals concur with current guidelines.

Setting: 4 major neuroscience centres in London. Participants: 58 consultants in emergency medicine and neuroscience specialities (neurology, neurosurgery and neuroradiology) participated in an anonymous survey.

\section{Primary and secondary outcome measures:}

The primary outcome measure was the highest stated acceptable risk of missing SAH in the neurologically intact patient presenting with sudden onset headache. Secondary outcome measures included agreement with guideline recommendations, risk/benefit appraisal and required performance of diagnostic tests, including lumbar puncture.

Results: Emergency department clinicians accepted almost 3 times the risk of a missed SAH diagnosis compared with the neuroscience specialists $(2.8 \%$ vs $1.1 \% ; p=0.02$ ), were more likely to accept a higher risk of missed diagnosis for the benefit of a non-invasive test $(p=0.04)$ and were more likely to disagree with current published guidelines stipulating the need for LP in all CT-negative cases ( $p=0.001)$.

Conclusions: Divergence from recognised procedures for SAH investigation is often criticised and attributed to a lack of knowledge of guidelines. This study indicates that divergence from guidelines may be explained by alternative risk-benefit appraisals made by doctors with their patients. Guideline recommendations may gain wider acceptance if they accommodate the requirements of the doctors and patients using them. Further study of clinical risk tolerance may help explain patterns of diagnostic test use and other variations in healthcare delivery.

\section{INTRODUCTION}

Controversies in the investigation of suspected spontaneous subarachnoid haemorrhage (SAH) principally concern the

\section{Strengths and limitations of this study}

- Novel survey addressing a controversial clinical scenario commonly encountered by all acute medical physicians.

- Explanation for variation in clinical practice that has been widely criticised and has a profound impact on patients, doctors and healthcare institutions.

- Pilot survey with a modest sample size $(n=58)$.

- No suitable validated instrument was available to measure clinicians' risk tolerance.

- Potential participation bias due to low response rate.

question of whether or not a lumbar puncture (LP) is needed following a normal CT scan. Missing the chance to treat acute aneurysmal SAH can have devastating consequences due to high associated mortality and morbidity. ${ }^{1}{ }^{2}$ Although an imperfect test, ${ }^{3}{ }^{4}$ the LP is held as a gold standard in the diagnosis of SAH, and when combined with CT has been shown to detect $\mathrm{SAH}$ reliably. ${ }^{5}$ Current international and national guidelines assert that SAH cannot be excluded based on a negative CT scan. Investigation is deemed incomplete unless negative imaging is followed by a lumbar puncture with spectrophotometric assessment of the supernatant for cerebrospinal fluid bilirubin. ${ }^{26-9}$

UK-based studies have found frequent deviation from guidelines. LP omission rates of $40-50 \%$ are common and are only slightly lower in teaching hospitals compared with District General Hospitals. ${ }^{10-13}$ UK figures are in line with international practice where rates of LP omission range between $40 \%$ and $60 \% .^{14-16}$

Although guidelines are not 'railroads' for clinicians to follow, ${ }^{17}$ omission of LP in 
suspected SAH diagnosis has been widely criticised, ${ }^{8} 101118$ and attributed to a lack of awareness and education. ${ }^{10}{ }^{14}$ However, the role of LP has been called into question due to reported low specificity, low clinical impact, low diagnostic yield and improved sensitivity of modern CT. ${ }^{3}{ }^{49-23}$ Doctors are also known to vary in their tolerance of risk and uncertainty which may influence management decisions. ${ }^{24-26}$ The low LP rate in suspected SAH may therefore represent widespread disagreement with guidelines and greater tolerance of the risk of misdiagnosis. A fear of LP-related complications may also dissuade clinicians from performing or recommending the procedure. ${ }^{21}$

Guidelines for SAH investigation have been shown to be relatively risk intolerant compared with recommended investigations of other potentially fatal conditions presenting to the emergency department (ED), for example, pulmonary embolism (PE) and acute coronary syndrome (ACS). Guidelines for suspected PE and ACS allow small but non-zero calculated risk end points, while SAH guidelines afford no misses. ${ }^{27}$ ED clinicians habituated to accept small risks of misdiagnosis for common emergency presentations (such as PE and ACS), may be more willing to accept similar risks in SAH work-up compared with their neurospeciality colleagues (ie, neurologists, neurosurgeons and neuroradiologists). Given that patients with suspected $\mathrm{SAH}$ are typically managed by emergency physicians, ${ }^{28}$ any such variation in risk appraisal could help explain the frequency of LP omission.

The purpose of this study is therefore twofold:

1. to survey clinicians' analysis of risk and benefit related to investigation of suspected acute spontaneous $\mathrm{SAH}$;

2. to establish whether published guidelines reflect current professional opinion, and to characterise any variation between ED clinicians and neurospecialists.

The null hypothesis is that ED clinicians are no more risk tolerant than neurospecialist consultants.

Given an LP omission rate of between $40 \%$ and $50 \%$ in the UK, at least $40 \%$ of ED clinicians are expected to disagree with the guideline stating that LP is required in CT-negative cases.

\section{METHODS}

No appropriate validated instruments were available to measure risk tolerance for missed SAH diagnosis. We therefore elected to design a questionnaire for this purpose according to principles described in detail elsewhere (see online supplementary material). ${ }^{29} 30$ The questionnaire underwent initial trial use by consultant and trainee clinicians in the relevant specialities. Changes were made to wording, question format and layout following this exercise.

Each question was devised to investigate clinicians' perceptions about aspects of the diagnostic process: clinical risk factors; LP complications; risk tolerance for misdiagnosis; sensitivity requirements of diagnostic tests; risk-benefit appraisal for diagnostic tests and investigative inertia, that is, the propensity to pursue a diagnosis due to referral bias rather than personal appraisal. While piloting the questionnaire, it became apparent that respondents could be identified if they provided their age, gender and speciality. Demographic details were therefore limited to include age range $(<40,41-50$, $51-60,>60)$ and speciality but not gender.

The primary outcome of risk tolerance was measured using a short vignette describing an otherwise well adult aged 40 years, presenting with a worst-ever 'thunderclap headache'. Participants were asked to give the post-test probability of missed SAH that they would accept before stopping further investigations. Respondents were told that the pretest probability for patients was $10 \%$, a conservative estimate and in line with similar clinical scenarios in the published literature. ${ }^{31}$

The primary objective of this study was to investigate patterns of risk tolerance and guideline agreement between neurospecialists and emergency physicians. A lack of validated instruments and relevant preliminary data prohibited a power calculation. We opted to sample two groups (neurospecialists and emergency physicians) comprising a quota target of 50 respondents, half from each group. Consultant neurosurgeons, neurologists, neuroradiologists and emergency clinicians were approached at four large NHS Trusts with tertiary neuroscience services: UCLH, Kings College Hospital, Queens Hospital Romford and Charing Cross/St Mary's Hospital. London Trusts were chosen to reduce the influence of regional variations.

A written questionnaire was devised in preference to an electronic survey because of concerns that electronic correspondence would be missed or ignored among the large volumes of information received electronically by NHS consultants.

Permission to conduct the survey was then sought from the clinical lead in each department. Invitations to participate were communicated by telephone, email and in person wherever possible in order to optimise participation. Secretarial staff were approached to help disseminate the questionnaires and email reminders were sent to eligible consultants where necessary. Data collection was carried out between October 2015 and February 2016.

\section{Statistical analysis}

Data were entered into an Excel spreadsheet (Excel for Mac, 2011). The significance of group differences was calculated using $\chi^{2}$ statistical tests within Excel. The statistical package (StatPlus) was used for descriptive statistics and for the t-test statistic where appropriate.

\section{Patient involvement}

No patients were involved in setting the research question or the outcome measures, nor were they involved in developing plans for recruitment, design or 
implementation of the study. No patients were asked to advise on interpretation or the writing up of results.

\section{RESULTS}

A total of 58 consultants completed the questionnaire, including $23 \mathrm{ED}$ clinicians and 35 neuroscience specialists. The overall response rate was $34 \%$ with proportional participation by both subgroups (35\% for the neurospecialists compared with $32 \%$ of ED physicians). The median age range was $41-50$ years and consultants had a median of 16-25 years of postgraduate experience. The gender of participants was not available for reasons described above. A total of 17 questions (1.5\%) were omitted or ignored by the 58 respondents. A further seven responses were excluded from analysis because they were incompatible with the question asked, indicating a misunderstanding or misreading.

Seventeen ED clinicians and 30 neurospecialists indicated their risk tolerance for missed SAH diagnosis by recording the highest post-test probability at which they would stop investigations to diagnose SAH (Question 10). There was a significant difference in the mean scores between groups $(p=0.03)$ with the ED clinicians' risk tolerance almost three times higher than the neurospecialists' $\quad(2.8 \% \quad(\mathrm{SD} \quad 3.3) \quad$ vs $1.1 \% \quad$ (SD 1.9$)$ ). Neurospecialists were also more likely to advocate routine LPs compared with ED clinicians ( $74 \%$ vs $39 \%$, $\mathrm{p}=0.01$ ).

Only $70 \%$ of consultants agreed with current guidelines stipulating that an LP is mandatory in suspected $\mathrm{SAH}$ when an initial CT is negative (table 1). There was a significant difference between the ED and neurospecialist groups in this regard with a majority of the ED group disagreeing with guidelines $(57 \%)$ compared with a minority $(11 \%)$ of neurospecialist consultants $(p<0.001)$. ED clinicians were more inclined to omit the LP if a negative CT had been obtained within 6 hours of headache onset ( $35 \%$ vs $3 \%, \mathrm{p}=0.002$ ).

ED consultants also required a higher pick-up rate to justify the routine use of LP compared with their neurospecialist colleagues, but this difference did not achieve statistical significance (table 2).

There was no significant group difference between the clinicians' personal experiences of LP-related complications with both groups median estimate falling in the 'infrequent' ( 1 in 200) category (figure 1).

Fewer than $10 \%$ of respondents in each group indicated a willingness to substitute the LP in favour of a cheaper or quicker test if it carried an increased risk of missed diagnosis. However, ED clinicians were more likely to accept an increased risk of misdiagnosis for the benefit of a non-invasive test $(38 \%$ vs $11 \%$, $\mathrm{p}=0.02$ ).

Almost all clinicians in both groups reported direct personal experience of missed SAH due to incomplete investigation (91\% in both groups). Fifty-five per cent of clinicians had given evidence in a medicolegal capacity and although this was more common among ED clinicians $(65 \%$ vs $55 \%)$, the difference was not statistically significant.

Local practices and policies were influential in deciding work-up of SAH; however, personal experience was the most commonly cited factor by clinicians (illustrated in figure 2). Nevertheless, $22 \%$ of clinicians reported that they would feel obliged to investigate SAH if it had been raised and documented as a potential diagnosis, irrespective of their own clinical judgement.

Although free text answers were not requested from participants, some responses were annotated. Where relevant, these are discussed below.

\section{DISCUSSION}

Although SAH is a relatively rare disease, its misdiagnosis was a familiar scenario for more than $90 \%$ of consultants. The consequence of missed diagnosis can be devastating for patients and carries significant medicolegal implications for doctors and healthcare institutions. It is therefore difficult to understand the frequency with which clinicians stray from recommended practice, especially considering the defensive nature of modern medicine. $^{32}$

There is an extensive body of published research evaluating the appropriateness of different strategies for $\mathrm{SAH}$ diagnosis. While there is some controversy regarding the utility of LP in the diagnostic pathway, current national and international guidelines unanimously recommend the procedure for patients with negative initial imaging. A recent survey of UK Emergency and Acute

Table 1 Agreement with guidelines

\begin{tabular}{llcc}
\hline Question/dimension & $\begin{array}{l}\text { All clinicians combined } \\
(\mathbf{n = 5 8 )}\end{array}$ & $\begin{array}{l}\text { ED physicians } \\
(\mathbf{n = 2 3 )}\end{array}$ & $\begin{array}{l}\text { Neurospecialists } \\
(\mathbf{n}=\mathbf{3 5})\end{array}$ \\
\hline LP mandatory 'No' & $17(30 \%)$ & $13(57 \%)^{*}$ & $4(11 \%)$ \\
LP mandatory 'Yes' & $40(70 \%)$ & $9(39 \%)^{*}$ & $31(89 \%)$ \\
LP omitted when CT <6 hours 'No' & $46(84 \%)$ & $15(65 \%)$ & $31(89 \%) \dagger$ \\
LP omitted when CT <6 hours 'Yes' & $9(16 \%)$ & $8(35 \%)$ & $1(3 \%) \dagger$ \\
\hline 'Question omitted by one consultant. & & & $\mathbf{0 . 0 0 0 1}$ \\
†Question omitted by three consultants. & & & \\
LP, lumbar puncture. & & &
\end{tabular}


Table 2 Clinicians' risk-benefit appraisals

\begin{tabular}{|c|c|c|c|c|}
\hline Question/dimension & $\begin{array}{l}\text { All clinicians combined } \\
(\mathrm{n}=58)\end{array}$ & $\begin{array}{l}\text { ED physicians } \\
(\mathrm{n}=23)\end{array}$ & $\begin{array}{l}\text { Neurospecialists } \\
(\mathrm{n}=\mathbf{3 5})\end{array}$ & p Value \\
\hline Routine LP justified? 'No' & $23(40 \%)$ & $14(61 \%)$ & $9(26 \%)$ & \\
\hline Routine LP justified? 'Yes' & $35(60 \%)$ & $9(39 \%)$ & $26(74 \%)$ & 0.007 \\
\hline Investigative inertia 'No' & $45(78 \%)$ & $20(87 \%)$ & $25(71 \%)$ & \\
\hline Investigative inertia 'Yes' & $13(22 \%)$ & $3(13 \%)$ & $10(29 \%)$ & 0.2 \\
\hline $\begin{array}{l}\text { Required LP pick-up rate: } \geq 1 \text { SAH every } \\
100 \text { LPs }\end{array}$ & $25(44 \%)$ & $12(52 \%)$ & $13(38 \%)^{*}$ & \\
\hline 1 SAH every $101-500$ LPs & $19(33 \%)$ & $8(35 \%)$ & $11(32 \%)^{*}$ & \\
\hline $1 \mathrm{SAH}$ for every $>500 \mathrm{LPs}$ & $13(23 \%)$ & $3(13 \%)$ & $10(29 \%)^{*}$ & 0.3 \\
\hline \multicolumn{5}{|l|}{ Risk-benefit trade off $†$} \\
\hline \multicolumn{5}{|l|}{ Non-invasive test } \\
\hline Yes & $12(21 \%)$ & $8(38 \%) \ddagger$ & $4(11 \%)$ & 0.018 \\
\hline No & $44(79 \%)$ & $13(62 \%) \ddagger$ & $31(89 \%)$ & \\
\hline \multicolumn{5}{|l|}{ Quicker test } \\
\hline Yes & $5(9 \%)$ & $2(10 \%) \ddagger$ & $3(9 \%)^{\star}$ & 0.9 \\
\hline No & $50(89 \%)$ & 19 (90\%)‡ & $31(89 \%)^{*}$ & \\
\hline \multicolumn{5}{|l|}{ Cheaper test } \\
\hline Yes & $5(9 \%)$ & $2(10 \%) \ddagger$ & $3(9 \%)^{*}$ & 0.9 \\
\hline No & $50(89 \%)$ & $19(90 \%) \ddagger$ & $31(89 \%)^{*}$ & \\
\hline Experience of previous missed SAH 'No' & $5(9 \%)$ & $2(9 \%)$ & $3(9 \%)^{\star}$ & \\
\hline Experience of previous missed SAH 'Yes' & $52(91 \%)$ & $21(91 \%)$ & $31(91 \%)^{*}$ & 0.9 \\
\hline
\end{tabular}

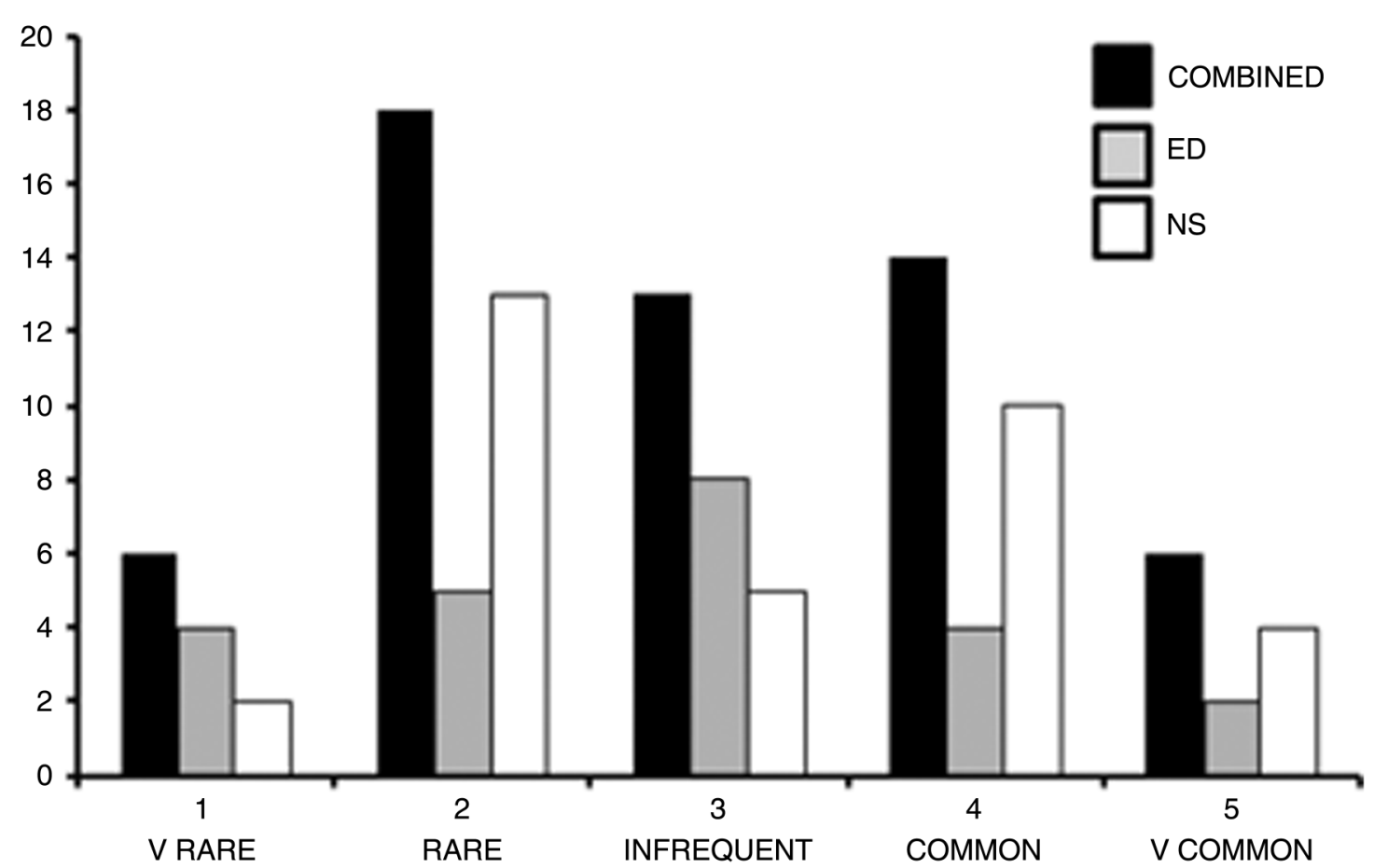

Figure 1 Clinicians' estimated frequency of lumbar puncture complications. Ordinal scale provided: Very rare is $<1$ in 200 ; Rare $\sim 1$ in 500; Infrequent 1 in 200; Common 1 in 100; Very common 1 in 20.

medicine clinicians confirms a wide variation in practice with only $74 \%$ of respondents following the recommended CT-LP pathway. ${ }^{33}$
To the best of our knowledge, this is the first study to investigate whether clinicians' risk tolerance might account for the observed variation in practice and to 
Figure 2 Factors reported to influence work up of subarachnoid haemorrhage.

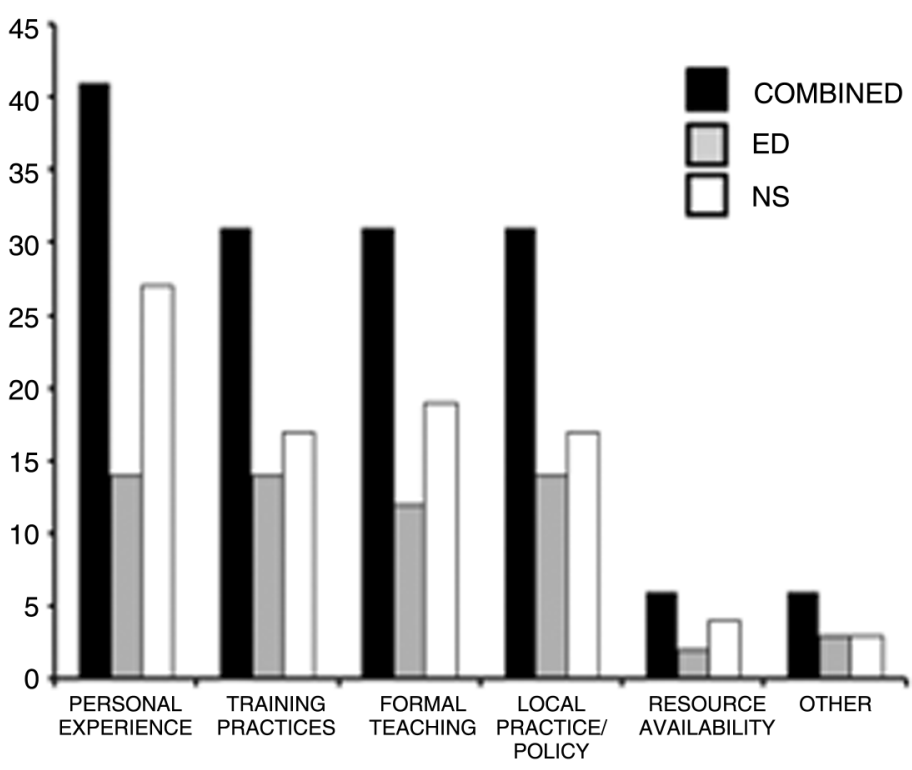

survey clinicians' appraisals of the relevant recommendations directly.

Almost all respondents had a personal experience of missed SAH diagnosis; however, only $70 \%$ agreed with the guidelines that LP was mandatory for all CT-negative cases. When ED clinicians were considered separately, only $39 \%$ held this view. Previous attempts to explain deviation from guidelines have suggested lack of awareness as a possible cause. Taken together, our findings suggest that clinicians are well aware of the perils of missed diagnosis, but are nevertheless sceptical of the benefits of LP in the diagnostic pathway.

It is typically the emergency and acute medical physicians who are compelled to perform an LP before referring or discharging patients with suspected SAH. Neurospecialists are more likely to be involved in an advisory capacity until a diagnosis has been made. The LP may therefore serve as a filter reducing unnecessary referral to specialist services. ${ }^{3}$ ED consultants were more willing to trade off benefits of a non-invasive diagnostic test against the increased risk of missed diagnosis. Neurospecialists did not tend to share this view, which may reflect a concern that referrals would increase if alternative tests were more readily performed.

This survey was not designed to test clinicians' knowledge of guidelines, epidemiology of SAH or the performance of diagnostic tests. Nevertheless, some responses did highlight common misconceptions, which are worth addressing here. Although the LP is taken as a gold standard test, the actual sensitivity in CT-negative SAH is unknown. ${ }^{28}$ The commonly quoted sensitivity of photospectrometry for xanthochromia comes from Vermeulen et $a l^{34}$ who reported $100 \%$ sensitivity up until 2 weeks after ictus $(n=32)$. These data were derived from LPs performed in patients with CT-positive SAH. ${ }^{34}$ The sensitivity of LP in CT-negative patients would be a more useful measure since this is the context in which the LP is employed. Unfortunately, this is difficult to quantify since there is no confirmatory test against which the LP can be measured.

The high frequency of equivocal photospectrometry findings is another shortcoming of the LP. In a large observational study by Sayer et $a l,{ }^{4}$ more than $15 \%$ of 1898 LPs were inconclusive by Clinical Biochemistry Guidelines. ${ }^{35}$ This large contemporary sample is arguably more representative of current clinical practice than the Vermulen cohort and demonstrates that even when applying the CT-LP paradigm, SAH cannot be entirely excluded. This may explain why ED clinicians are less likely to consider the LP as a prerequisite for SAH diagnosis.

Recent research has suggested that CT approaches $100 \%$ sensitivity in acute $\mathrm{SAH}$ if performed within 6 hours of ictus. This has led to calls for a change of practice. ${ }^{36-39}$ Participants were specifically asked about this scenario. ED clinicians were more willing to adopt this policy than their neurospecialist counterparts, which may reflect a desire to reduce pressure on EDs.

The concept of safe discharge has been deemed 'irrational unhelpful and unachievable' because it raises unrealistic expectations of a risk-free environment. ${ }^{40}$ Conversely, accepting an arbitrary threshold for risk may seem callous or even negligent as it consigns some patients to an adverse outcome. ${ }^{41}$ Interestingly, five neurospecialists stated that they would only stop investigations for $\mathrm{SAH}$ when the risk of missed diagnosis was reduced to zero. None of the ED consultants shared this view. ED clinicians' reluctance to resort to LP may therefore reflect the greater tolerance of risk in this group.

It is arguably inappropriate-for patients and health services-that a medical intervention should be determined entirely by the risk threshold of an individual doctor. $^{41}$ However, it is equally undesirable for guidelines to impose an arbitrary risk threshold that is based 
on opinion rather than evidence. To be accepted by clinicians and patients, guidelines may need to accommodate a range of possible risk tolerance preferences. This may apply to a variety of clinical situations-involving different specialities and healthcare systems-where there is a fine balance between clinical risk and benefit.

Our findings suggest that the current guidelines do not closely reflect ED clinicians' risk tolerance for SAH investigation-despite the fact they usually manage patients presenting with the acute sudden onset headache. Incidentally, the Royal College of Emergency Medicine was not represented in the formulation of the current RCP Stroke guidelines (confirmed by personal correspondence).

\section{Limitations of this study}

The lack of an appropriate instrument to measure clinicians' risk tolerance necessitated the development of a novel questionnaire. Despite our best efforts, the responses indicated some shortcomings of this tool that limit the strength of our data. Seven respondents were known to have misinterpreted questions because the answers they provided were incompatible with the question asked. Although these responses could be excluded from the final analysis, other misrepresentative responses may still exist within the data set. The development and validation of an instrument to reliably measure risk tolerance was outside the scope of this study. Although we did not feel the lack of such a tool precluded investigation, our findings require cautious interpretation as a result.

A targeted approach to recruitment (by email, telephone or in person) was adopted to optimise participation. However, this may have introduced a degree of bias since not all respondents were contacted in exactly the same manner or at the same time. An online survey may have generated more responses and would have been easier to conduct at a national level but would not have overcome participation bias. We felt a targeted pilot survey was an appropriate starting point that could be used to inform future study.

The survey is limited by a poor response rate, which introduces the possibility of participation bias. Although response rates were similar between the two groups, different factors may have influenced each group. Response rates were not balanced across institutions (ranging between $17 \%$ and $42 \%$ ) and as such, our results are skewed towards the NHS Trust that provided the most responses.

As with any survey of this type, hypothetical scenarios can never accurately reflect real-life practice. This problem is exaggerated when considering abstract concepts such as risk. We asked respondents to quantify their acceptable risk of missed diagnosis. This is a particularly challenging task-reflected in the fact that four consultants did not provide a response. Clinicians may have felt uncomfortable characterising their clinical judgement in statistical terms. However, this is a common scenario faced by clinicians in their daily practice when patients ask them to quantify the chance of complications from an invasive procedure, diagnostic test or surgery. Furthermore, incorporating relevant quantitative research findings into clinical decisionmaking is necessary to deliver evidence-based medicine (EBM). Therefore, although a simulation, the scenario we presented to clinicians is a realistic challenge similar to that commonly faced in the healthcare setting.

One respondent indicated that they would ask the patient to determine their own level of acceptable risk rather than assume this responsibility for themselves. It is possible that other participants shared a similar view and felt our question was incompatible with their own professional approach. This raises interesting questions about the doctor-patient relationship, the didactic nature of guidelines and the role of patients in guideline development. Clearly, clinical risks need to be acceptable to patients first and foremost and patient centredness has become a dominant paradigm in modern medicine. ${ }^{42}$ However, transferring responsibility to the patient can only lead to empowerment if patients are supported in the decision-making process and the best evidence is made easily accessible to them.

Patient participation is encouraged in guideline development ${ }^{43}$ on the assumption that active patient involvement will enhance the quality of guidelines. However, it has been argued that this mechanism is ill suited to achieving patient-centred care at the individual level. As described by van de Bovenkamp and Trappenburg, ${ }^{42}$ training and supporting patients to participate as full members in guideline development is a double-edged sword. Patients who have been adequately trained and supported become fellow academics and may no longer be able to contribute the experiential knowledge for which they were asked to participate in the first place. Patients who were not properly trained do contribute this experiential knowledge, but studies have shown that it is difficult to incorporate this in EBM guidelines.

Clinicians continue to play a key role in guiding patients through complex medical decisions. Although challenging, further study of clinicians' risk-benefit appraisals could improve our understanding of clinical behaviours and ensure that guidelines and doctors work together to deliver patient-centred care.

Knowledge of clinicians' risk tolerance could also inform diagnostic test development, given that the usefulness of a test is determined by more than its accuracy alone. For example, our findings suggest that some clinicians would prefer a non-invasive alternative to LP, despite a lower sensitivity. Conversely, a diagnostic test offering improved accuracy may fail to gain acceptance if other characteristics were less tolerable to doctors and patients. This is borne out by the growing use of noninvasive vascular imaging techniques in SAH diagnosis. Considerable advances in non-invasive vascular imaging methods such as CT angiography (CTA) and MR angiography (MRA) have led to their use in clinical practice. A CT/CTA diagnostic paradigm has been reported to have 
a sensitivity of $99 \%$ for aneurysmal SAH diagnosis and has been proposed as a substitute for LP. ${ }^{44}$ Opponents criticise this approach for moving from the diagnosis of haemorrhage to the detection of aneurysms, which may be coincidental. ${ }^{45}$ Nevertheless, given the advantages afforded by non-invasive tests, the use of CTA and MRA may need to be incorporated into future guidelines for the management of patients with suspected SAH.

\section{Conclusion}

This study provides a possible explanation for the observed variation in practice of SAH investigation. Opinions vary significantly between ED clinicians and neurospecialists with respect to the utility of LP and their tolerances for the risk of missed diagnosis. Omission of the LP from the diagnostic work-up may reflect scepticism about its utility rather than an unawareness of the risk of missed diagnosis.

Guidelines provide essential, expert evidence summaries and can marshal clinicians through a complex and ever-changing scientific literature. Nevertheless, guidelines, healthcare institutions, clinicians and patients may continue to contradict each other when they are informed by different tolerances of risk. Guidelines may be used more widely if they accommodate different preferences where appropriate.

Future study of risk tolerance in the clinical setting may enhance our understanding of a range of clinical behaviours, including referral patterns and diagnostic test use. Development of validated instruments to measure risk tolerance is needed, so that robust conclusions can be drawn from future research.

\section{Author affiliations}

${ }^{1}$ UCL Institute of Neurology, National Hospital for Neurology and

Neurosurgery, Queen Square, London, UK

${ }^{2}$ Barts Health NHS Trust, London, UK

${ }^{3}$ Education Unit, UCL Institute of Neurology, National Hospital for Neurology and Neurosurgery, Queen Square, London, UK

${ }^{4}$ Imperial Health NHS Trust, London, UK

${ }^{5}$ Neuroradiological Academic Unit, Department of Brain Repair and Rehabilitation, UCL Institute of Neurology, Queen Square, London, UK

Acknowledgements Zoe Fox provided assistance with statistical analysis.

Contributors $\mathrm{JL}$ contributes to design, data collection, analysis and manuscript preparation. CS contributes to design, analysis, manuscript preparation and editing. HRJ contributes to design, manuscript preparation. AK contributes to manuscript preparation. $\mathrm{KL}$ contributes to manuscript preparation.

Funding This research received no specific grant from any funding agency in the public, commercial or not-for-profit sectors. MD-Res tuition fees for JL were paid for by the Royal College of Radiologists Kodak Radiology Fund Scholarship.

\section{Competing interests None declared.}

Ethics approval UCL Research Ethics commitee.

Provenance and peer review Not commissioned; externally peer reviewed.

Data sharing statement No additional data are available.

Open Access This is an Open Access article distributed in accordance with the Creative Commons Attribution Non Commercial (CC BY-NC 4.0) license, which permits others to distribute, remix, adapt, build upon this work noncommercially, and license their derivative works on different terms, provided the original work is properly cited and the use is non-commercial. See: http:// creativecommons.org/licenses/by-nc/4.0/

\section{REFERENCES}

1. Roos YB, de Haan RJ, Beenen LF, et al. Complications and outcome in patients with aneurysmal subarachnoid haemorrhage: a prospective hospital based cohort study in the Netherlands. J Neurol Neurosurg Psychiatr 2000;68:337-41.

2. Connolly ES Jr, Rabinstein AA, Carhuapoma JR, et al. Guidelines for the management of aneurysmal subarachnoid hemorrhage: a guideline for healthcare professionals from the American Heart Association/American Stroke Association. Stroke 2012;43:1711-37.

3. Ditta M, Galea J, Holland J, et al. Lumbar puncture and the diagnosis of CT negative subarachnoid haemorrhage: time for a new approach? Br J Neurosurg 2013;27:599-602.

4. Sayer D, Bloom B, Fernando K, et al. An observational study of 2,248 patients presenting with headache, suggestive of subarachnoid hemorrhage, who received lumbar punctures following normal computed tomography of the head. Acad Emerg Med 2015;22:1267-73.

5. Perry JJ, Spacek A, Forbes M, et al. Is the combination of negative computed tomography result and negative lumbar puncture result sufficient to rule out subarachnoid hemorrhage? Ann Emerg Med 2008;51:707-13.

6. Intercollegiate Stroke Working Party. National clinical guideline for stroke, 4th edition. London: Royal College of Physicians Physicians, 2012.

7. Vivancos J, Gilo F, Frutos R, et al. Clinical management guidelines for subarachnoid haemorrhage. Diagnosis and treatment. Neurologia 2014;29:353-70.

8. Steiner T, Juvela S, Unterberg A, et al. European Stroke Organization guidelines for the management of intracranial aneurysms and subarachnoid haemorrhage. Cerebrovasc Dis 2013;35:93-112.

9. Scottish Intercollegiate Guidelines Network, Diagnosis and management of headache in adults. A national clinical guideline. Edinburgh: Scottish Intercollegiate Guidelines Network (SIGN), 2008.

10. Schofield ML, Lorenz E, Hodgson TJ, et al. How well do we investigate patients with suspected subarachnoid haemorrhage? The continuing need for cerebrospinal fluid investigations. Postgrad Med J 2004;80:27-30.

11. Mehrotra P, Sookhoo S, Kolla S, et al. Investigation of subarachnoid haemorrhage: does the buck stop with CT? J Med Life 2010;3:338-42.

12. O'Neill J, McLaggan S, Gibson R. Acute headache and subarachnoid haemorrhage: a retrospective review of CT and lumbar puncture findings. Scott Med J 2005;50:151-3.

13. Cooper JG, Smith B, Hassan TB. A retrospective review of sudden onset severe headache and subarachnoid haemorrhage on the clinical decision unit: looking for a needle in a haystack? Eur $J$ Emerg Med 2016;23:356-62.

14. Muhammed O, Teubner D, Jones DN, et al. Retrospective audit of the investigation of patients with suspected acute subarachnoid haemorrhage. J Med Imaging Radiat Oncol 2010;54:339-46.

15. Morgenstern LB, Luna-Gonzales H, Huber JC, Jr., et al. Worst headache and subarachnoid hemorrhage: prospective, modern computed tomography and spinal fluid analysis. Ann Emerg Med 1998;32(Pt 1):297-304.

16. Perry JJ, Eagles D, Clement CM, et al. An international study of emergency physicians' practice for acute headache management and the need for a clinical decision rule. CJEM 2009;11:516-22.

17. Tingle JH. Do guidelines have legal implications? Arch Dis Child 2002;86:387-8.

18. Martin SC, Teo MK, Young AM, et al. Defending a traditional practice in the modern era: the use of lumbar puncture in the investigation of subarachnoid haemorrhage. Br J Neurosurg 2015;29:799-803.

19. Brunell A, Ridefelt $P$, Zelano J. Differential diagnostic yield of lumbar puncture in investigation of suspected subarachnoid haemorrhage: a retrospective study. J Neurol 2013;260:1631-6.

20. Wood MJ, Dimeski G, Nowitzke AM. CSF spectrophotometry in the diagnosis and exclusion of spontaneous subarachnoid haemorrhage. J Clin Neurosci 2005;12:142-6.

21. Migdal VL, Wu WK, Long D, et al. Risk-benefit analysis of lumbar puncture to evaluate for nontraumatic subarachnoid hemorrhage in adult ED patients. Am J Emerg Med 2015;33:1597-601. 
22. Gee C, Dawson M, Bledsoe J, et al. Sensitivity of newer-generation computed tomography scanners for subarachnoid hemorrhage: a Bayesian analysis. J Emerg Med 2012;43:13-18.

23. Boesiger BM, Shiber JR. Subarachnoid hemorrhage diagnosis by computed tomography and lumbar puncture: are fifth generation CT scanners better at identifying subarachnoid hemorrhage? J Emerg Med 2005;29:23-7.

24. Holtgrave DR, Lawler F, Spann SJ. Physicians' risk attitudes, laboratory usage, and referral decisions: the case of an academic family practice center. Med Decis Making 1991;11:125-30.

25. Pines JM, Hollander JE, Isserman JA, et al. The association between physician risk tolerance and imaging use in abdominal pain. Am J Emerg Med 2009;27:552-7.

26. Pines JM, Isserman JA, Szyld D, et al. The effect of physician risk tolerance and the presence of an observation unit on decision making for ED patients with chest pain. Am J Emerg Med 2010;28:771-9.

27. Pines JM, Szyld D. Risk tolerance for the exclusion of potentially life-threatening diseases in the ED. Am J Emerg Med 2007;25:540-4

28. Davenport R. Diagnosing acute headache. Clin Med (Lond) 2004;4:108-12.

29. Boynton PM, Greenhalgh T. Selecting, designing, and developing your questionnaire. BMJ 2004;328:1312-15.

30. Rattray J, Jones MC. Essential elements of questionnaire design and development. J Clin Nurs 2007;16:234-43.

31. Edlow JA, Wyer PC. How good is a negative cranial computed tomographic scan result in excluding subarachnoid hemorrhage? Ann Emerg Med 2000;36:507-16.

32. Anderson RE. Billions for defense: the pervasive nature of defensive medicine. Arch Intern Med 1999;159:2399-402.

33. Dobb B, Cooper J. A pilot survey of decisions by acute medicine staff after thunderclap headache. $J R$ Coll Physicians Edinb 2013;43:207-14.

34. Vermeulen M, Hasan D, Blijenberg BG, et al. Xanthochromia after subarachnoid haemorrhage needs no revisitation. J Neurol Neurosurg Psychiatr 1989;52:826-8.
35. Cruickshank A, Auld P, Beetham R, et al. Revised national guidelines for analysis of cerebrospinal fluid for bilirubin in suspected subarachnoid haemorrhage. Ann Clin Biochem 2008;45(Pt 3):238-44

36. Edlow JA, Fisher J. Diagnosis of subarachnoid hemorrhage: time to change the guidelines? Stroke 2012;43:2031-2.

37. Backes D, Rinkel GJ, Kemperman H, et al. Time-dependent test characteristics of head computed tomography in patients suspected of nontraumatic subarachnoid hemorrhage. Stroke 2012;43:2115-19.

38. Rinkel GJ. Management of patients with aneurysmal subarachnoid haemorrhage. Curr Opin Neurol 2016;29:37-41.

39. Blok KM, Rinkel GJ, Majoie CB, et al. CT within 6 hours of headache onset to rule out subarachnoid hemorrhage in nonacademic hospitals. Neurology 2015;84:1927-32.

40. Goodacre S. Safe discharge: an irrational, unhelpful and unachievable concept. Emerg Med J 2006;23:753-5.

41. O'Keeffe ST. A cross-sectional study of doctors', managers' and public representatives' views regarding acceptable level of risk in discharges from the emergency department. QJM 2015;108:533-8.

42. van de Bovenkamp HM, Trappenburg MJ. Reconsidering patient participation in guideline development. Health Care Anal 2009;17:198-216.

43. National Institute for Health and Care Excellence. NICE process and methods guides. Developing NICE Guidelines: the manual. London: National Institute for Health and Care Excellence (NICE), 2015.

44. McCormack RF, Hutson A. Can computed tomography angiography of the brain replace lumbar puncture in the evaluation of acute-onset headache after a negative noncontrast cranial computed tomography scan? Acad Emerg Med 2010;17:444-51.

45. Edlow JA. What are the unintended consequences of changing the diagnostic paradigm for subarachnoid hemorrhage after brain computed tomography to computed tomographic angiography in place of lumbar puncture? Acad Emerg Med 2010;17:991-5; discussion 96-7. 